\title{
OPTICAL SPECTRA OF CANDIDATE SOUTHERN HEMISPHERE INTERNATIONAL CELESTIAL REFERENCE FRAME (ICRF) RADIO SOURCES
}

\author{
O. Titov ${ }^{1}$, D. L. Jauncey ${ }^{2}$, H. M. Johnston ${ }^{3}$, R. W. Hunstead ${ }^{3}$, And L. Christensen ${ }^{4}$ \\ ${ }^{1}$ Geoscience Australia, P.O. Box 378, Canberra, ACT 2601, Australia; oleg.titov@ga.gov.au \\ ${ }^{2}$ CSIRO Astronomy and Space Science, ATNF \& Mount Stromlo Observatory, Cotter Road, Weston, ACT 2611, Australia \\ ${ }^{3}$ Sydney Institute for Astronomy, School of Physics, University of Sydney, NSW 2006, Australia \\ ${ }^{4}$ Technische Universität Munich, Excellence Cluster Universe, Boltzmannstr. 2, D-85748 Garching, Germany \\ Received 2011 July 11; accepted 2011 September 3; published 2011 October 17
}

\begin{abstract}
We present the results of spectroscopic observations of the optical counterparts of 47 southern radio sources from the candidate International Celestial Reference Catalogue as part of a very long baseline interferometry (VLBI) program to strengthen the celestial reference frame, especially in the south. We made the observations with the $3.58 \mathrm{~m}$ European Southern Observatory New Technology Telescope. We obtained redshifts for 30 quasars and one radio galaxy, with a further seven objects being probable BL Lac objects with featureless spectra. Of the remainder, four were clear misidentifications with Galactic stars and five had low signal-to-noise spectra and could not be classified. These results, in combination with new VLBI data of the radio sources with redshifts more than 2, add significantly to the existing data needed to refine the distribution of source proper motions over the celestial sphere.
\end{abstract}

Key words: galaxies: distances and redshifts - quasars: emission lines - radio continuum: general - reference systems

Online-only material: color figures

\section{INTRODUCTION}

We investigate the intrinsic properties and distribution of the ultra-compact, flat-spectrum radio sources that make up the International Very Long Baseline Interferometry (VLBI) Service (IVS) Reference Catalogue. Astrometric VLBI measures precise group delays and the difference in arrival times of the radio waves at widely separated radio telescopes, and thus produces accurate radio positions typically with milliarcsecond precision. The first systematic astrometric VLBI program was started in 1979 and, since 2000, IVS has organized a comprehensive international program of precision position measurements of about 1000 sources (Schlüter \& Behrend 2007).

About 50 radio telescopes have participated in observations over the 30 year IVS history. Conventional reductions of highprecision multi-frequency VLBI data are made in the reference system with its origin at the barycenter of the solar system (McCarthy \& Petit 2004). This barycentric reference system was adopted by the International Astronomical Union (IAU) as the International Celestial Reference System (ICRS). By definition, the axes of the ICRS are fixed by the positions of selected extragalactic radio sources, and the reference frame representing the system should show no global rotation with respect to these sources. VLBI presently represents the most precise, practical, and reliable method for realizing such a catalog.

From 1998 through 2009 the initial International Celestial Reference Frame (ICRF1) was based on a catalog of 608 radio VLBI source positions. Of those, 212 were so-called defining sources that were used to establish the orientation of the ICRS axes (Ma et al. 1998). In 2009 the second realization of the ICRF (ICRF2) was put forward. ICRF2 contains 295 "defining" sources of which only 97 were "defining" in the ICRF1. The "inflated" floor error for the most observed radio source is reported as $41 \mu$ as (Fey et al. 2009), based on comparison of two astrometric catalogs obtained with two independent subsets of original VLBI data. Even for the radio sources with fewer observations, the median position error for the 1217 ICRF 2 radio sources (295 "defining" and 922 "non-defining") was found to be $174 \mu$ as in right ascension and $194 \mu$ as in declination. Thus, the errors of the radio catalogs are much less than the optical ones (see Section 4).

A separate program is underway to link the optical and radio reference frames, and one of the important tasks here is to make reliable optical identifications whenever an optical counterpart can be found. The European mission Gaia to be launched in 2013 will observe about 1 billion stars and about 500,000 quasars down to magnitude 20 (Perryman et al. 2001), with an accuracy of a few tens of $\mu$ as at magnitude 15-18 (Lindegren et al. 2008). The Gaia-based optical realization of the ICRF will be used in parallel with the current radio realization (ICRF2). Alignment between the two realizations will be an important issue. Particularly, only comparatively bright quasars $(m \leqslant 18)$ with stable astrometric positions are to be selected for statistical comparison. Bourda et al. (2008) showed that only 70 of the ICRF1 sources $(\sim 10 \%)$ are suitable for establishing the link. A dedicated project has started to increase the number of appropriate quasars among the ICRF2 radio sources (Bourda et al. 2011).

The total number of radio sources included in the IVS astrometric program exceeds 4000 , though only $\sim 1000$ sources are observed on a regular basis. The database of the radio source physical characteristics (Titov \& Malkin 2009) comprises 4261 objects (by 2010 August), mostly quasars, but there is a serious deficit in the southern hemisphere of candidate sources for which VLBI observations have been made. There is also a significant lack of optical identifications for the existing candidate radio sources. By 2010 July, of the 2211 ICRF2 sources with measured redshifts, only 781 are in the southern hemisphere and only 129 have declinations south of $-40^{\circ}$. Lack of redshifts in the south can cause difficulties in the analysis of apparent proper motions of the reference radio sources (Titov \& Malkin 2009). The newly identified quasars with $z \geqslant 2$ will be the subject of intensive VLBI observations using radio telescopes in the southern hemisphere. Additional astrometric positions will improve their proper motion estimates. Six quasars (B0008-300, B0034-220, B1505-304, 
B2107-105, B2318-087, and B2321-065) have been scheduled for observations in the latter part of 2011.

Our spectroscopic program therefore focuses on spectroscopic observations of the IVS radio sources with optical counterparts, especially those in the south as well as those with a long observational history. In this paper we present spectra of 44 extragalactic objects observed at the European Southern Observatory's (ESO) $3.58 \mathrm{~m}$ New Technology Telescope (NTT). We describe the data reduction and analysis procedures in Section 2 and report our results in Section 3. Throughout the paper we adopt the source names used by the IVS community; these are similar - and, in many cases, identical — to the original PKS B1950 convention.

\section{OBSERVATIONS}

The observations were carried out in 2010 August at the NTT (Visitor Mode run 085A-0588 (A)) using the ESO Faint Object Spectrograph and Camera (EFOSC) system with grism 13 covering the wavelength range $3685-9315 \AA$. The seeing was typically $0.8-1.5$ arcsec but occasionally as high as 4 arcsec. The measured spectral resolution was $21 \AA$ FWHM. After setting up on each target we observed for an initial 15 minutes, followed by an additional 15 minutes if no obvious emission line was seen on the first exposure; individual spectra were later combined. Wavelength calibration was performed using the spectrum of a $\mathrm{He} / \mathrm{Ne} / \mathrm{Ar}$ comparison lamp, resulting in typical fit errors of $0.5 \AA$ (rms).

We performed data reduction with the IRAF software suite using standard procedures for spectral analysis. We removed the bias and pixel-to-pixel gain variations from each frame and then removed cosmic rays using the IRAF task SZAP. The separate exposures were then combined and a single spectrum extracted. We calibrated the resulting one-dimensional spectrum in wavelengths and flux-calibrated each spectrum by comparing it with the spectrum of a spectrophotometric standard taken with the same instrumental setup. Because the observing conditions were not photometric, the flux calibration should be taken as approximate.

\section{RESULTS}

The outcomes from the spectroscopic observations of 47 targets were as follows (Table 1).

1. Emission-line redshifts: 31

2. Probable BL Lac objects: 8

3. Galactic stars: 3

4. Spectra with signal-to-noise ratio $(\mathrm{S} / \mathrm{N})$ too low to classify: 5 .

Spectra for 44 of the targets are presented in Figure 1, and emission-line data for the 31 objects for which redshifts have been determined are given in Table 2 . The radio positions were taken from the ICRF2 catalog (Fey et al. 2009).

Seven objects-IVS B0122-260, B1443-162, B1533-316, B1633-810, B2012-017, B2053-323, and B2254-204were found to have featureless spectra and hence are identified as probable BL Lac objects.

Four objects that were found close to the radio position were identified as stars. Two of these, IVS B1748-253 and IVS B1822-173, are within $3^{\circ}$ of the Galactic plane, are in dense star fields, and exhibit extinction in $V$ of at least $5 \mathrm{mag}$, so it remains unlikely that their actual optical counterparts will be detectable. Radio-optical position differences for all four objects are given in Table 3, and the identification is rejected on positional grounds in each case. A more detailed discussion
Table 1

Outcomes of the Spectroscopic Observations

\begin{tabular}{|c|c|c|c|}
\hline Source & R.A. $(J 2000)^{a}$ & Decl. $(\mathrm{J} 2000)^{\mathrm{a}}$ & Redshift \\
\hline IVS B0002-170 & 000517.933 & -164804.678 & $0.7804 \pm 0.0013$ \\
\hline IVS B0008-300 & 001045.177 & -294513.177 & $2.3439 \pm 0.0052$ \\
\hline IVS B0028-396 & 003124.331 & -392249.391 & $1.2978 \pm 0.0023$ \\
\hline IVS B0034-220 & 003714.825 & -214524.714 & $2.5133 \pm 0.0021$ \\
\hline IVS B0055-059 & 005805.066 & -053952.277 & $1.2456 \pm 0.0008$ \\
\hline IVS B0107-610 & 010915.475 & -604948.460 & Low S/N \\
\hline IVS B0110-668 & 011218.912 & -663445.187 & $1.1888 \pm 0.0021$ \\
\hline IVS B0122-260 & 012518.837 & -254904.390 & BL Lac \\
\hline IVS B0221-171 & 022343.763 & -165637.701 & $1.0152 \pm 0.0017$ \\
\hline IVS B1443-162 & 144553.376 & -162901.619 & BL Lac \\
\hline IVS B1452-168 & 145502.811 & -170013.953 & Low $\mathrm{S} / \mathrm{N}$ \\
\hline IVS B1505-304 & 150852.993 & -303629.430 & $3.3684 \pm 0.0023$ \\
\hline IVS B1511-476 & 151440.024 & -474829.858 & $1.5512 \pm 0.0018$ \\
\hline IVS B1533-316 & 153654.498 & -315115.135 & BL Lac \\
\hline IVS B1633-810 & 164257.346 & -810835.070 & BL Lac \\
\hline IVS B1635-141 & 163845.284 & -141550.237 & $0.2575 \pm 0.0004$ \\
\hline IVS B1647-296 & 165039.544 & -294346.955 & Low $\mathrm{S} / \mathrm{N}$ \\
\hline IVS B1659-621 & 170336.541 & -621240.008 & $1.7547 \pm 0.0012$ \\
\hline IVS B1707-038 & 171017.205 & -035550.128 & $1.9231 \pm 0.0017$ \\
\hline IVS B1726-038 & 172850.235 & -035050.436 & $0.6617 \pm 0.0003$ \\
\hline IVS B1748-253 & 175151.263 & -252400.064 & Star \\
\hline IVS B1758-651 & 180323.496 & -650736.761 & $1.1991 \pm 0.0006$ \\
\hline IVS B1815-553 & 181945.399 & -552120.745 & $1.6292 \pm 0.0013$ \\
\hline IVS B1822-173 & 182536.532 & -171849.848 & Star \\
\hline IVS B1852-534 & 185700.452 & -532500.356 & $0.7779 \pm 0.0007$ \\
\hline IVS B1923+210 & 192559.605 & +210626.162 & BL Lac \\
\hline IVS B1928-698 & 193331.159 & -694258.914 & $1.4807 \pm 0.0020$ \\
\hline IVS B1936-623 & 194121.769 & -621121.056 & Low $\mathrm{S} / \mathrm{N}$ \\
\hline IVS B2012-017 & 201515.158 & -013732.560 & BL Lac \\
\hline IVS B2053-323 & 205625.070 & -320847.801 & BL Lac \\
\hline IVS B2059-786 & 210544.961 & -782534.547 & Low $\mathrm{S} / \mathrm{N}$ \\
\hline IVS B2107-105 & 211000.978 & -102057.319 & $2.5004 \pm 0.0012$ \\
\hline IVS B2117-614 & 212104.074 & -611124.624 & $1.0168 \pm 0.0011$ \\
\hline IVS B2135-184 & 213841.928 & -181044.371 & $0.1887 \pm 0.0001$ \\
\hline IVS B2158-167 & 220054.878 & -163232.701 & $0.8355 \pm 0.0024$ \\
\hline IVS B2211-388 & 221438.569 & -383545.008 & $0.3888 \pm 0.0004$ \\
\hline IVS B2220-163 & 222341.172 & -160705.188 & $0.8811 \pm 0.0002$ \\
\hline IVS B2234-253 & 223718.355 & -250632.519 & $1.2788 \pm 0.0011$ \\
\hline IVS B2236-572 & 223912.075 & -570100.839 & $0.5686 \pm 0.0023$ \\
\hline IVS B2239-631 & 224307.839 & -625057.322 & $0.3924 \pm 0.0005$ \\
\hline IVS B2254-204 & 225641.208 & -201140.510 & BL Lac \\
\hline IVS B2300-307 & 230305.821 & -303011.473 & Star \\
\hline IVS B2318-087 & 232118.250 & -082721.521 & $3.1639 \pm 0.0033$ \\
\hline IVS B2321-065 & 232339.113 & -061759.238 & $2.1440 \pm 0.0020$ \\
\hline IVS B2327-459 & 233037.680 & -453958.101 & $0.4471 \pm 0.0004$ \\
\hline IVS B2344-514 & 234719.864 & -511036.065 & $1.7502 \pm 0.0060$ \\
\hline IVS B2354-251 & 235723.850 & -245103.163 & $1.6137 \pm 0.0014$ \\
\hline
\end{tabular}

Note. ${ }^{\text {a }}$ ICRF2 radio position.

of two special cases, IVS B1923 + 210 and IVS B2300-307, is given in subsections 3.2 and 3.3, respectively.

Finally, there were five targets with low-S/N spectra that could not be classified-IVS B0107-610, B1452-168, B1647-296, B1936-623, and B2059-786-due to either their faintness or poor observing conditions. The field of IVS B1647-296, at $\ell=352.5, b=9.35$, is too crowded to be confident that the object observed was the correct identification and that the spectrum was not contaminated by light from a neighboring star.

\subsection{Notes on Individual Sources}

1. IVS B0002-170-reported as a quasar with redshift $z=$ $0.77539(Q=3)$ in the $6 \mathrm{dF}$ survey (Jones et al. 2009); our 
IVS B0002-170 $z=0.780$
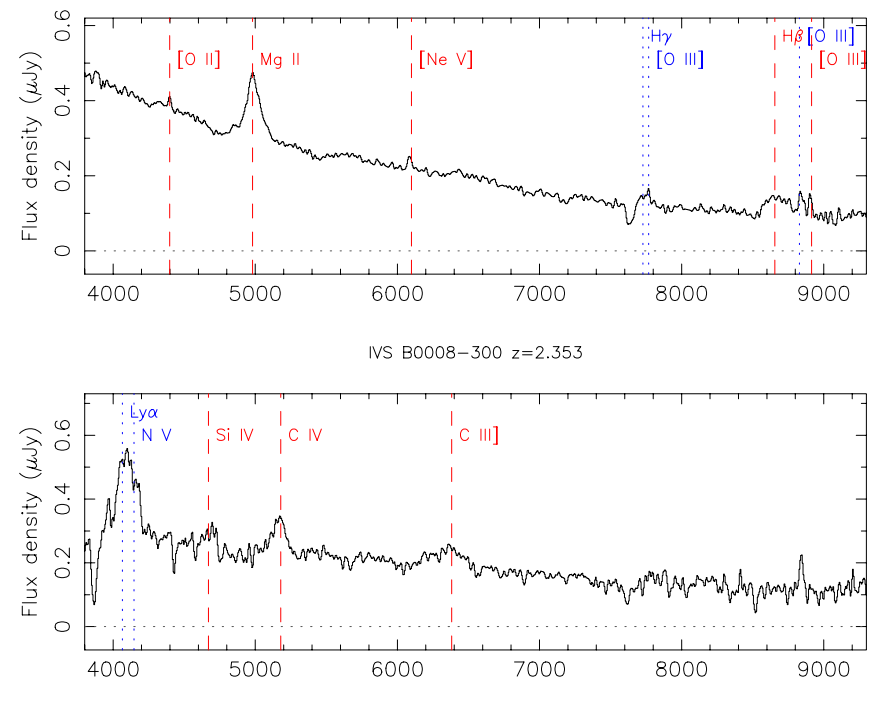

IVS B0028-396 $z=1.298$

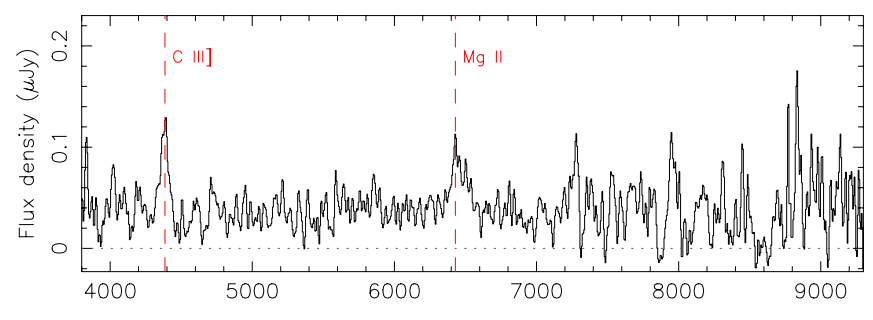

IVS B0034-220 $z=2.513$
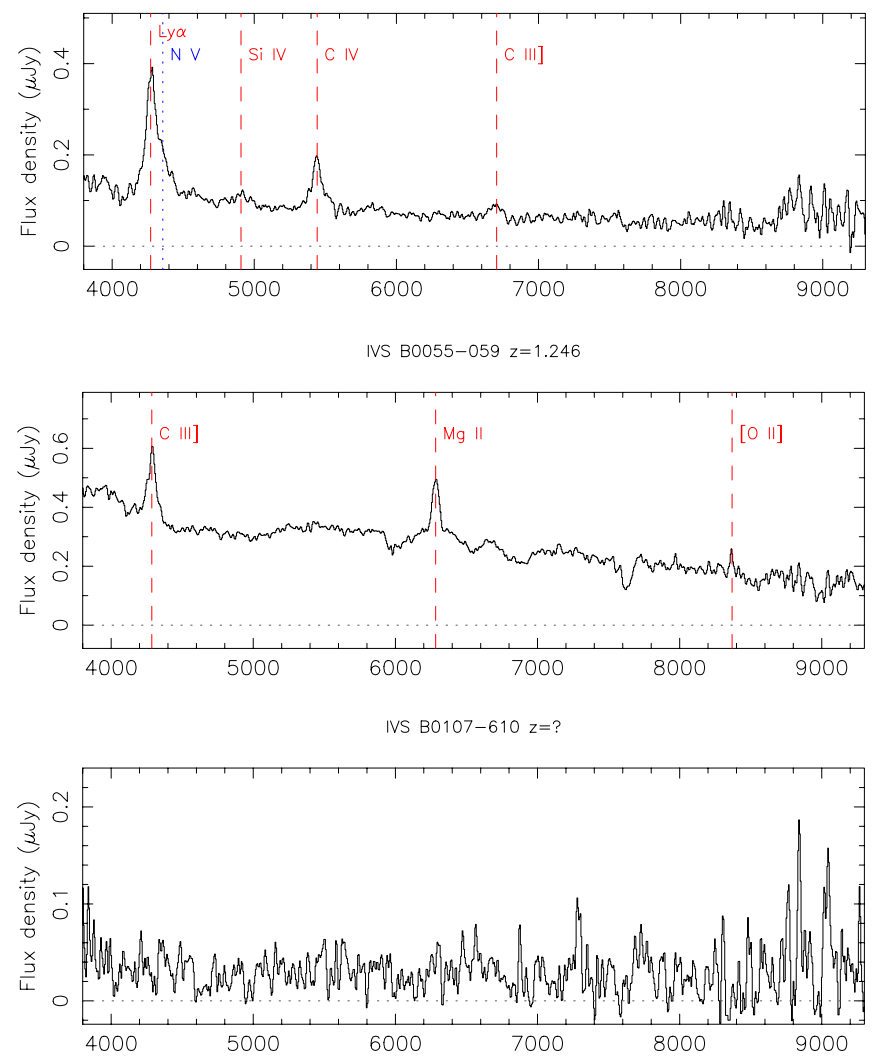

IVS B0221-171 $z=1.015$

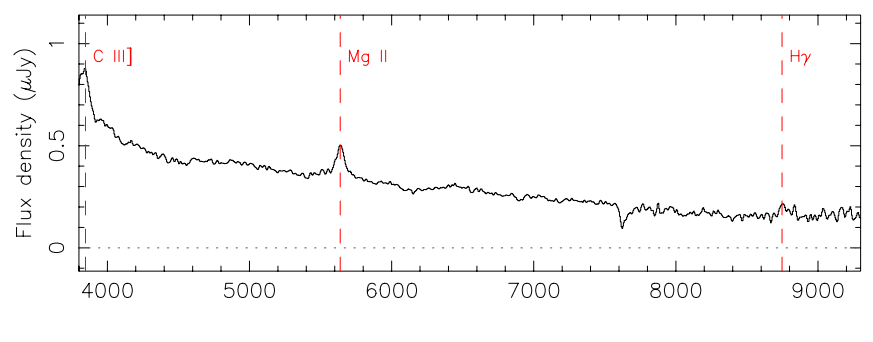

IVS B1443-162 BLL

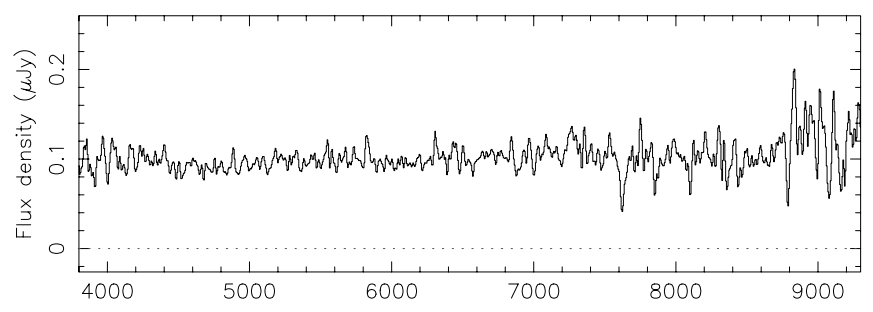

IVS B1452-168 $z=$ ?

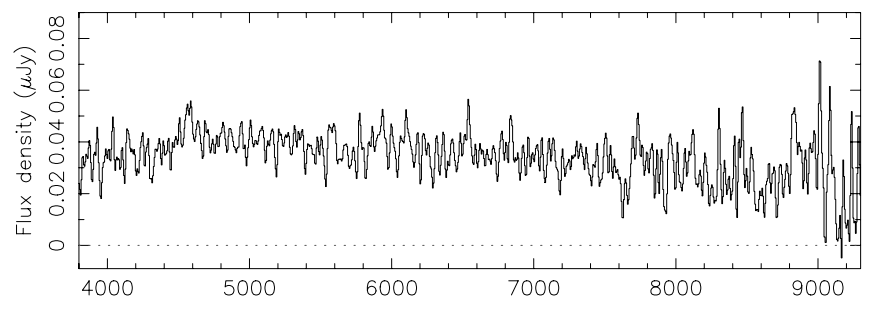

IVS $B 1505-304 \quad z=3.400$

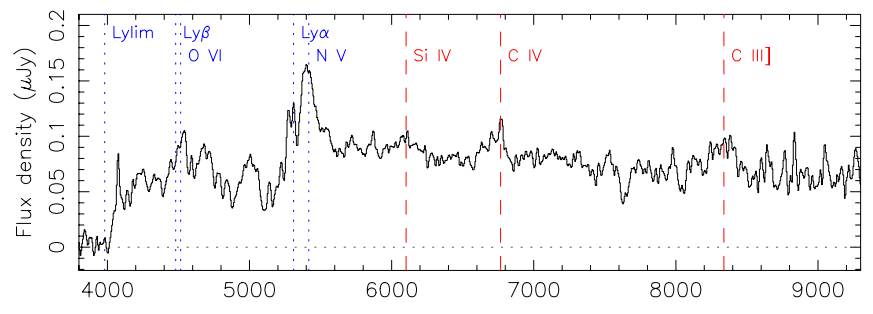

VS B1511-476 z=1.551
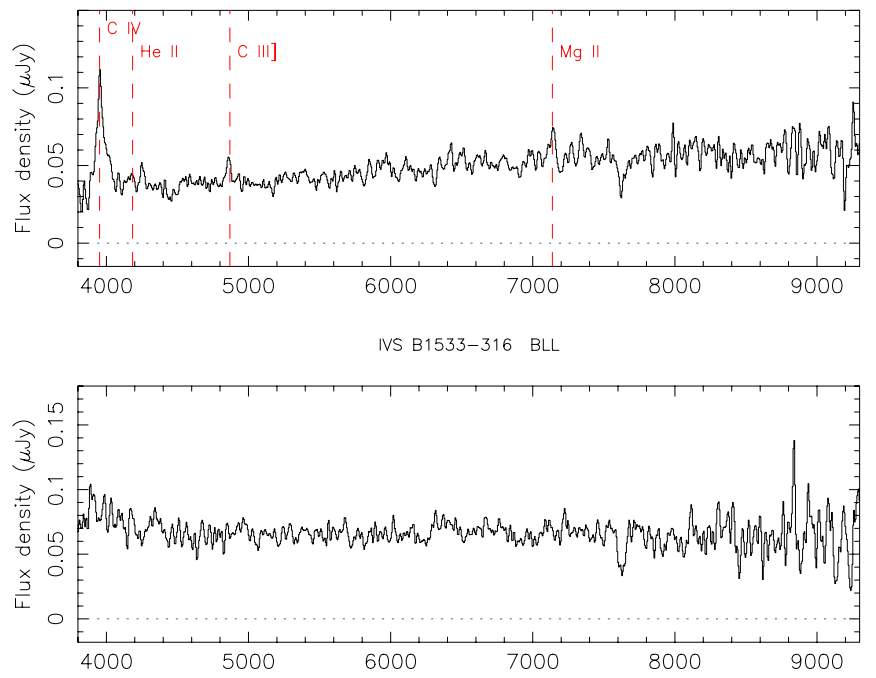

Figure 1. Optical spectra for 44 IVS targets, including 31 emission-line objects listed in Table 2, eight possible BL Lac objects, and five low signal-to-noise spectra that could not be classified. Dashed lines indicate emission lines used for redshift determination; dotted lines indicate features that were not used.

(A color version of this figure is available in the online journal.) 
IVS B0110-668 $z=1.189$

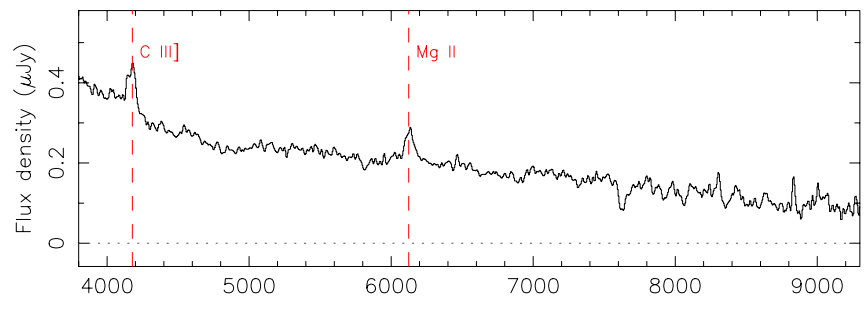

IVS B0122-260 BLL

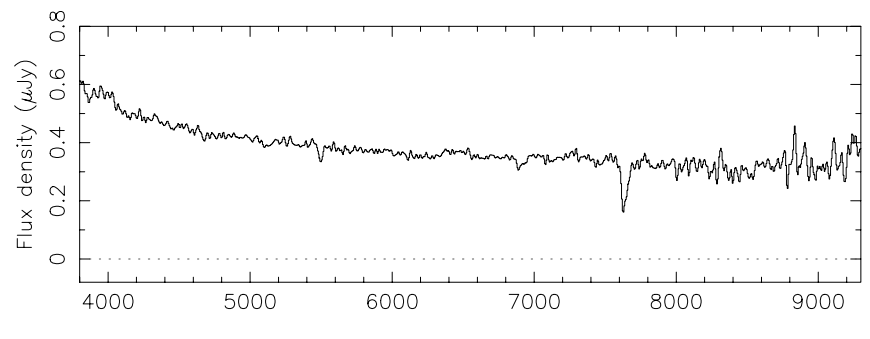

IVS B1647-296 $z=$ ?

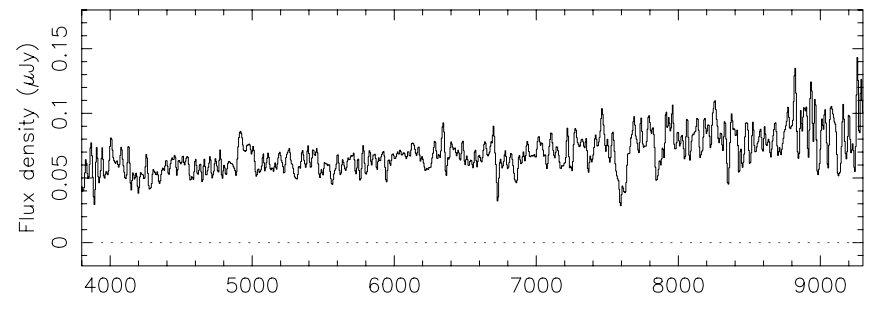

IVS B1659-621 $z=1.755$

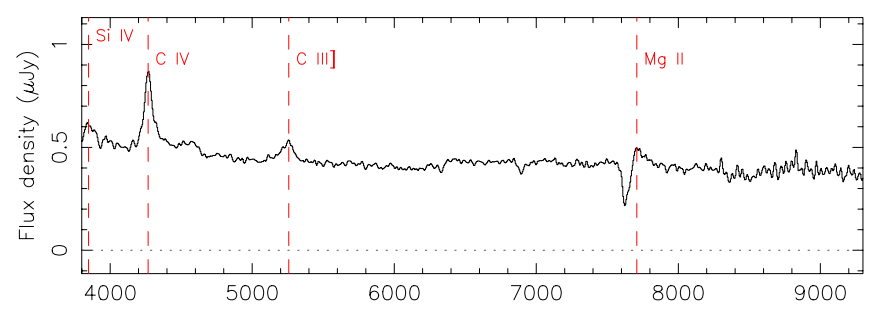

IVS B1707-038 $z=1.923$

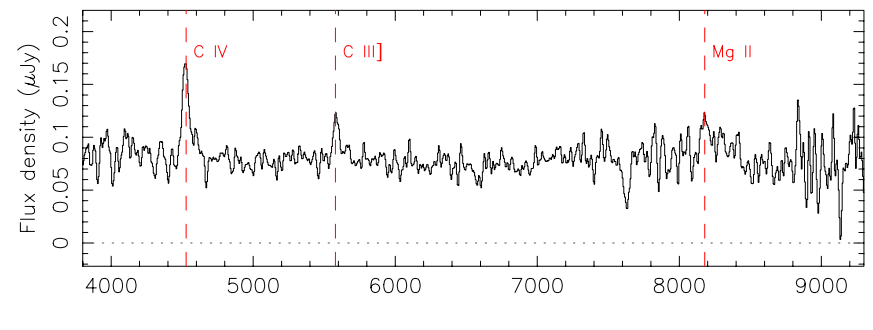

IVS B1726-038 $z=0.662$

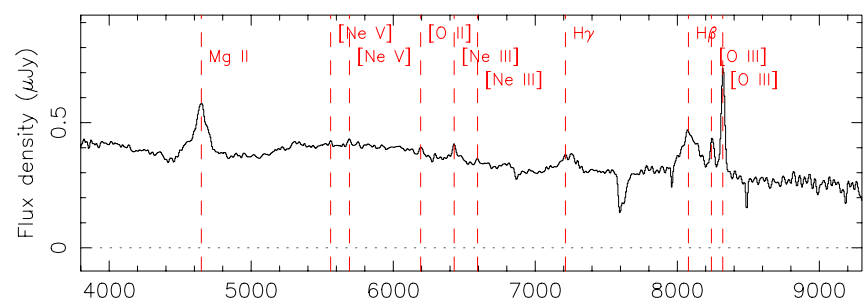

IVS B1633-810 BLL

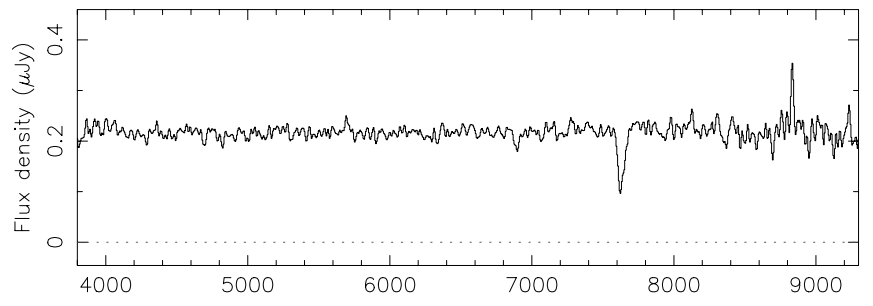

IVS B1635-141 $z=0.257$

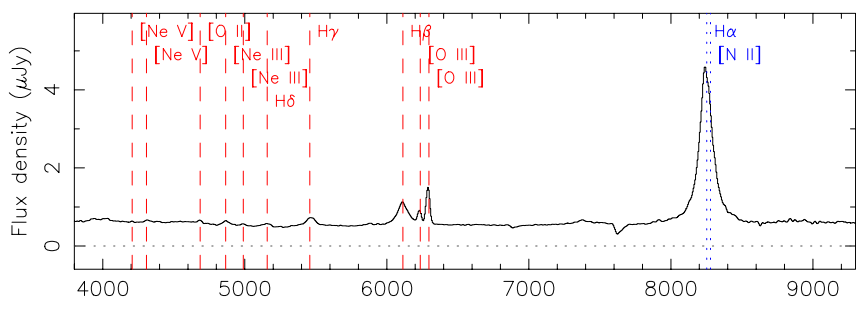

IVS B1928-698 $z=1.479$

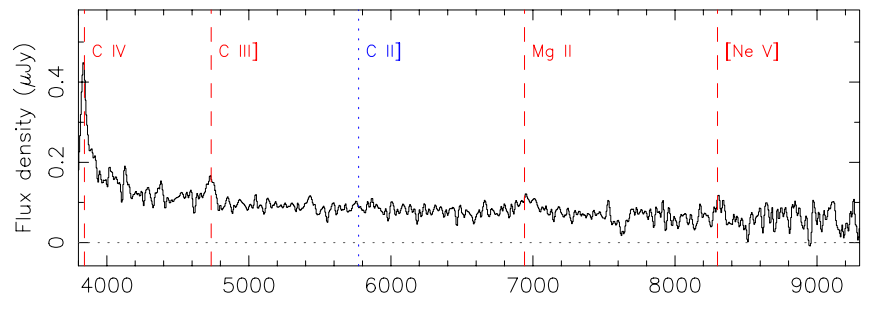

IVS B1936-623 z=?

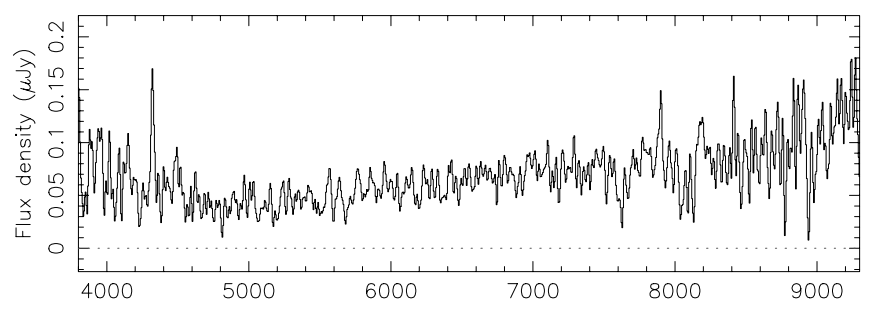

IVS B2012-017 BLL

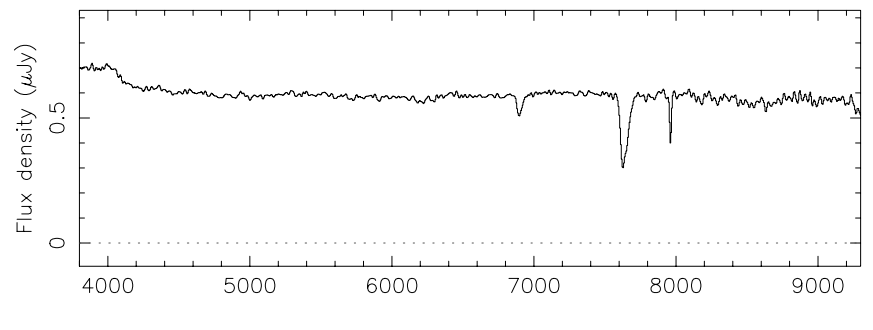

IVS B2053-323 BLL

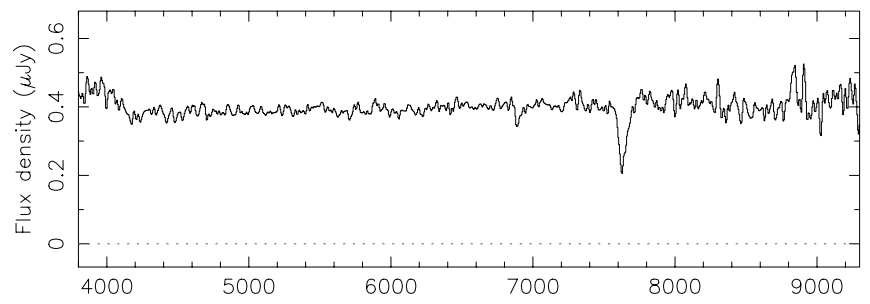

Figure 1. (Continued) 
IVS B1758-651 $z=1.198$

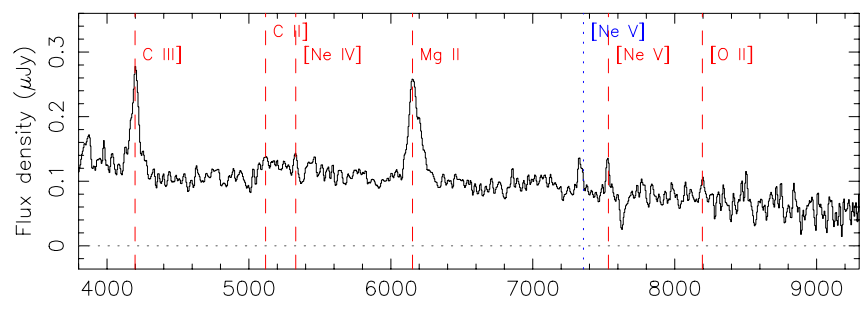

IVS B1815-553 $z=1.632$

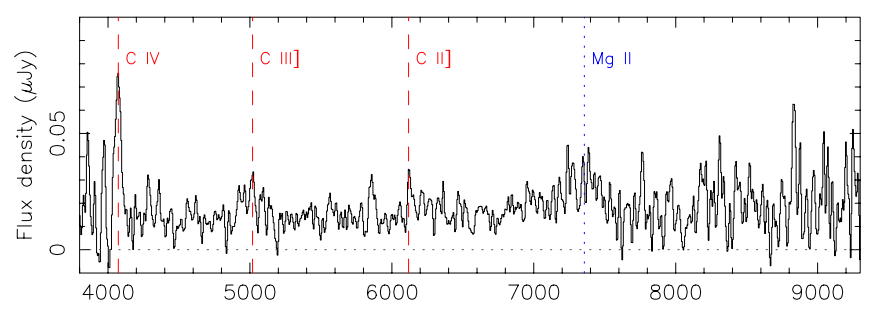

IVS B1852-534 $z=0.778$

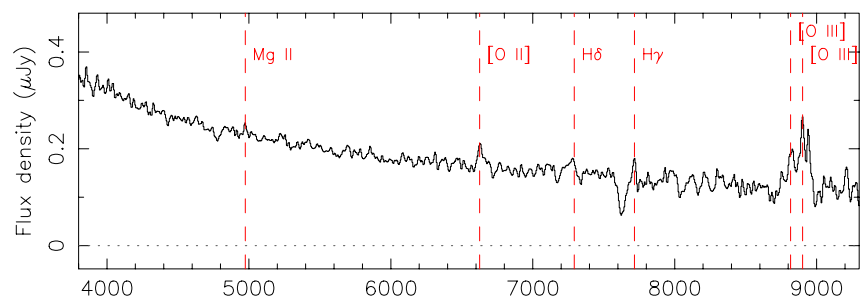

IVS B1923+210 BLL

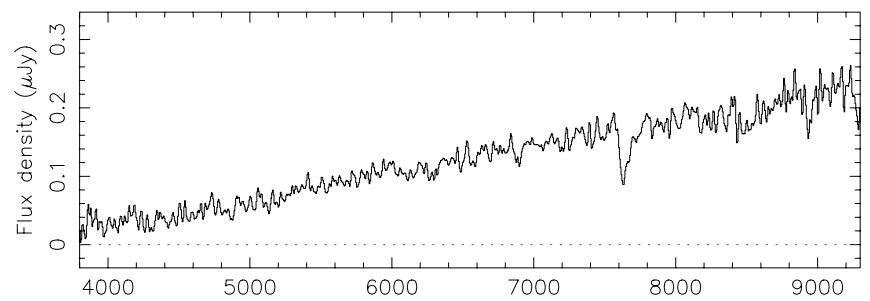

IVS B2158-167 $z=0.836$

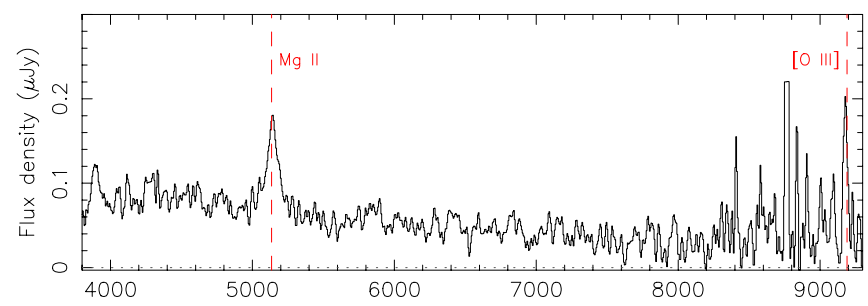

IVS B2211-388 $z=0.389$

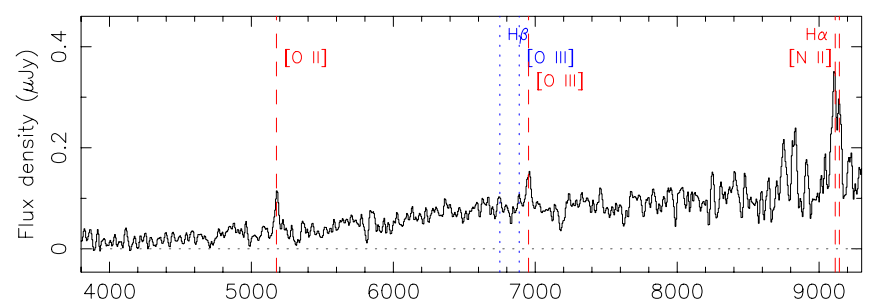

IVS B2059-786 $z=$ ?

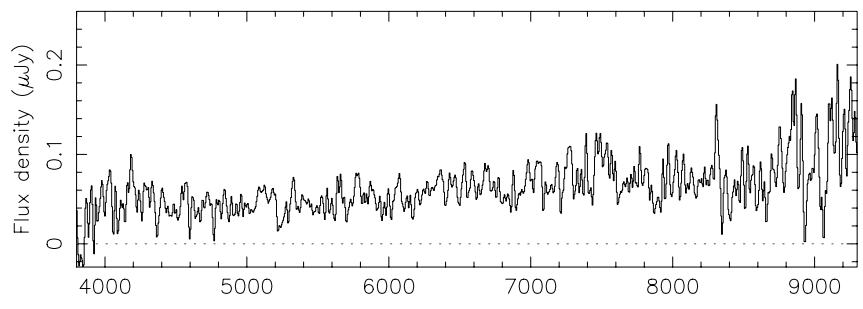

IVS B2107-105 $z=2.501$

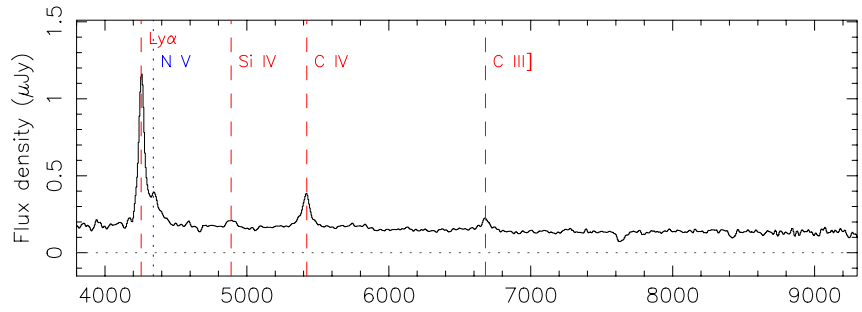

IVS B2117-614 $z=1.017$

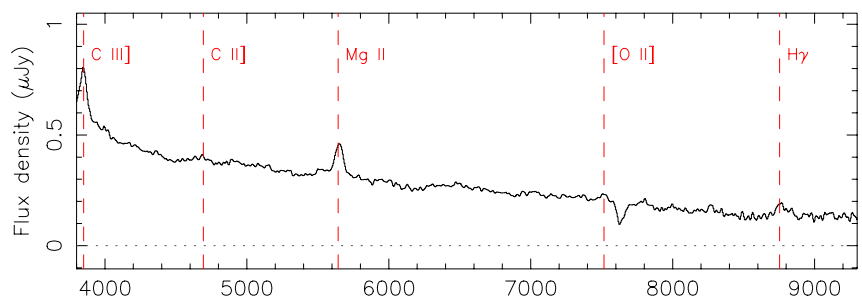

IVS B2135-184 $z=0.189$

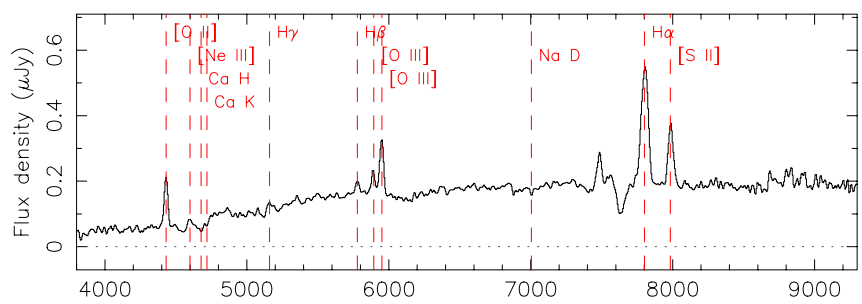

IVS B2236-572 $z=0.569$

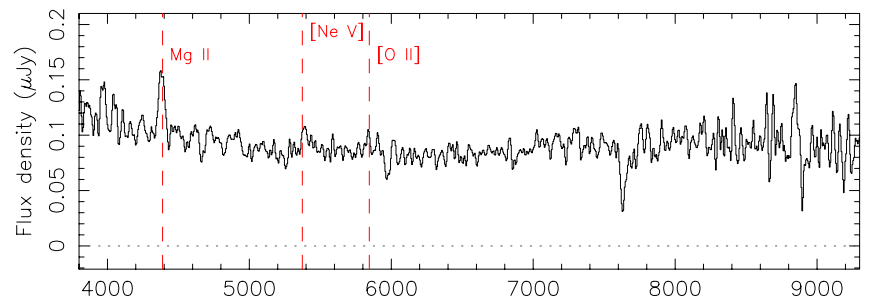

IVS B2239-631 $z=0.392$

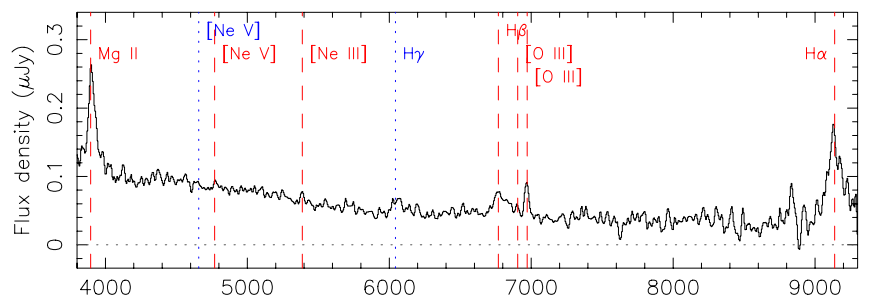

Figure 1. (Continued) 
IVS B2220-163 $z=0.882$
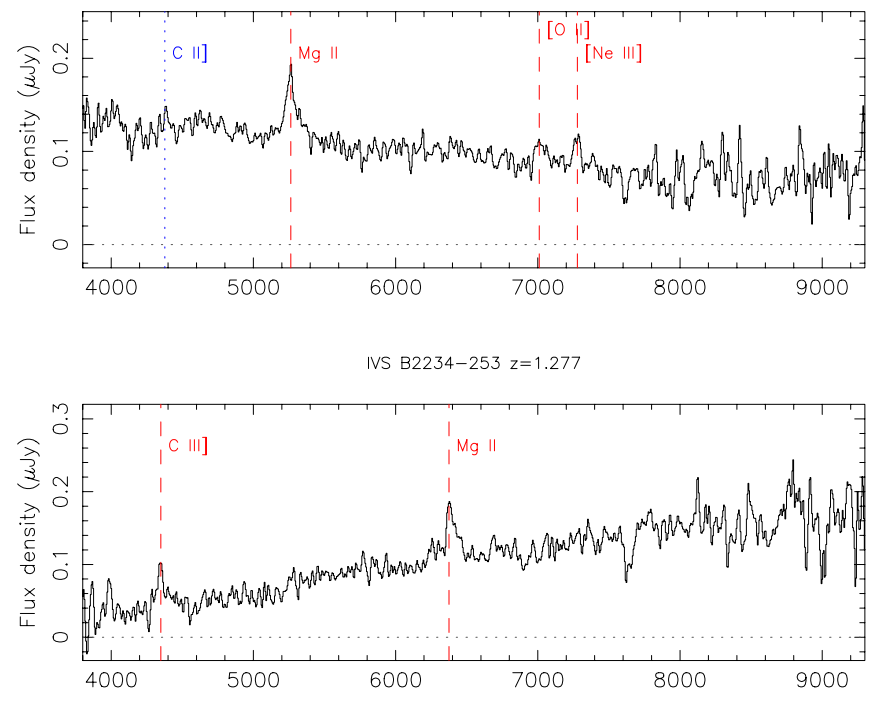

IVS B2254-204 BLL

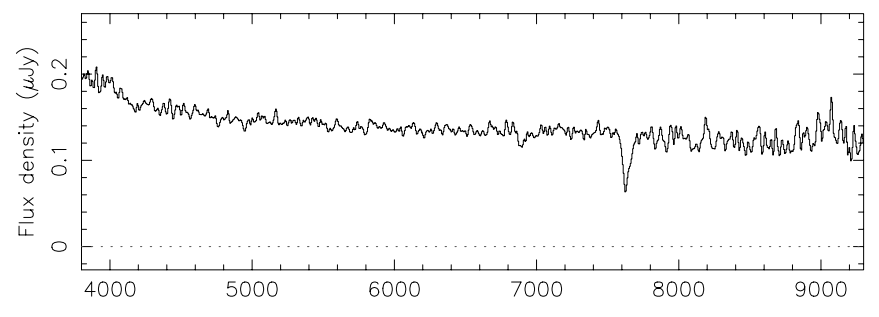

IVS B2318-087 $z=3.169$

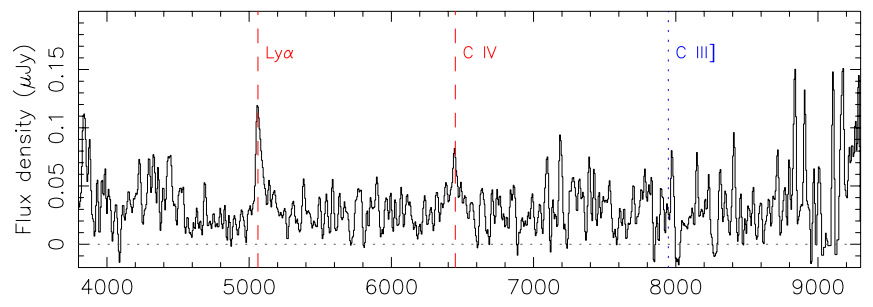

IVS B2321-065 $z=2.144$

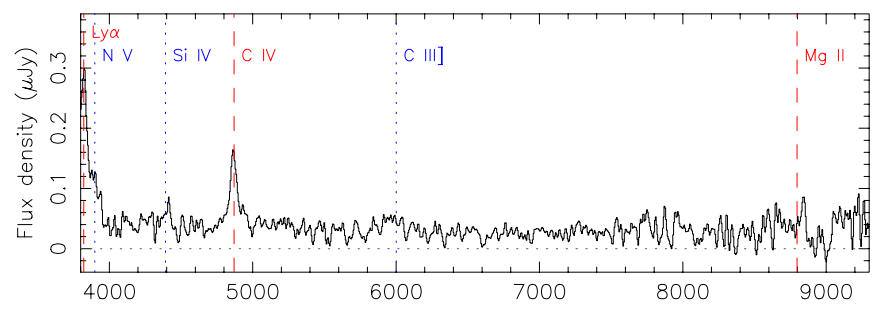

IVS B2327-459 $z=0.447$

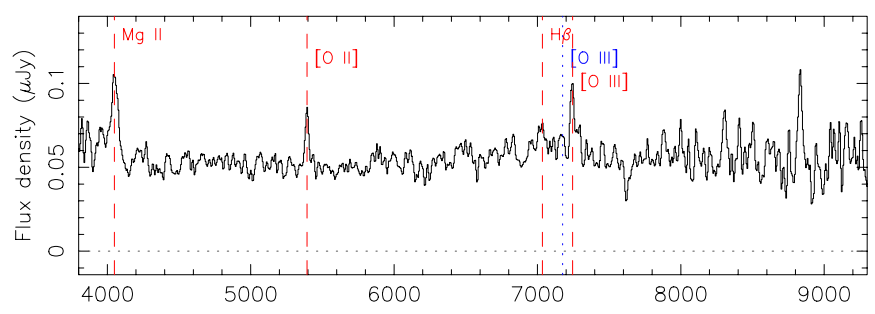

IVS B2344-514 $z=1.756$

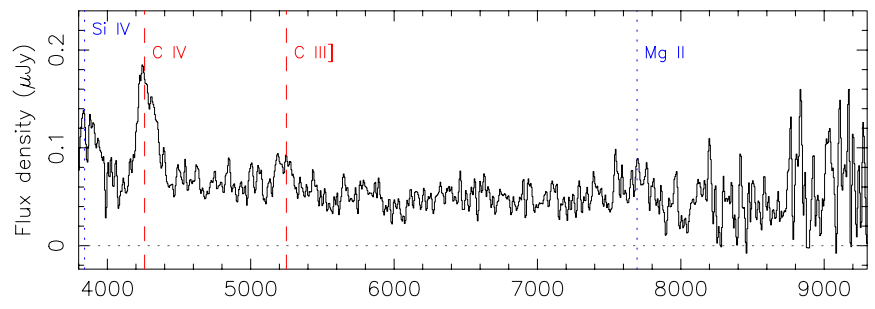

IVS B2354-251 $z=1.614$

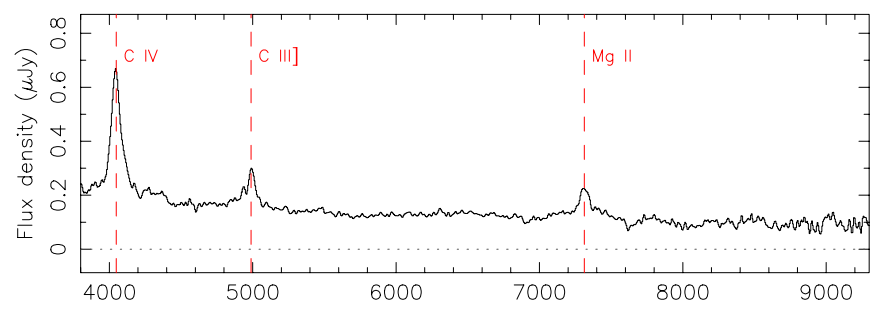

Figure 1. (Continued) 
Table 2

Observed Emission Lines

\begin{tabular}{|c|c|c|c|c|c|}
\hline Source & Line & $\begin{array}{c}\lambda_{\text {rest }} \\
(\AA)\end{array}$ & $\begin{array}{l}\lambda_{\text {obs }} \\
(\AA)^{\mathrm{a}}\end{array}$ & $z$ & Mean $z$ \\
\hline \multirow[t]{8}{*}{ IVS B0002-170 } & [O II $]$ & 2470.2 & 4398.0 & 0.7804 & $0.7804 \pm 0.0013$ \\
\hline & Mg II & 2797.9 & 4986.9 & 0.7824 & \\
\hline & {$[\mathrm{Ne} \mathrm{v}]$} & 3425.5 & 6086.4 & 0.7768 & \\
\hline & $\mathrm{H} \gamma$ & 4340.5 & 7715.7 & 0.7776 & \\
\hline & [O III] & 4363.2 & 7765.4 & 0.7797 & \\
\hline & $\mathrm{H} \beta$ & 4861.3 & 8673.7 & 0.7842 & \\
\hline & [O III] & 4958.9 & 8840.4 & 0.7827 & \\
\hline & [O III $]$ & 5006.8 & 8904.4 & 0.7784 & \\
\hline \multirow[t]{5}{*}{ IVS B0008-300 } & $\operatorname{Ly} \alpha$ & 1215.7 & 4090.0 & 2.3644 & $2.3439 \pm 0.0052$ \\
\hline & $\mathrm{Nv}$ & 1240.1 & 4176.4 & 2.3677 & \\
\hline & Si IV & 1396.8 & 4683.9 & 2.3534 & \\
\hline & C IV & 1549.1 & 5167.0 & 2.3356 & \\
\hline & C III] & 1908.7 & 6380.1 & 2.3426 & \\
\hline \multirow[t]{2}{*}{ IVS B0028-396 } & $\mathrm{C}$ III] & 1908.7 & 4381.4 & 1.2955 & $1.2978 \pm 0.0023$ \\
\hline & Mg II & 2797.9 & 6435.4 & 1.3001 & \\
\hline \multirow[t]{5}{*}{ IVS B0034-220 } & $\operatorname{Ly} \alpha$ & 1215.7 & 4276.2 & 2.5176 & $2.5133 \pm 0.0021$ \\
\hline & $\mathrm{N} \mathrm{v}$ & 1240.1 & 4355.3 & 2.5120 & \\
\hline & Si IV & 1396.8 & 4909.6 & 2.5150 & \\
\hline & C IV & 1549.1 & 5441.7 & 2.5129 & \\
\hline & $\mathrm{C}$ III] & 1908.7 & 6695.6 & 2.5079 & \\
\hline \multirow[t]{3}{*}{ IVS B0055-059 } & $\mathrm{C}$ III] & 1908.7 & 4287.5 & 1.2463 & $1.2456 \pm 0.0008$ \\
\hline & $\mathrm{Mg}$ II & 2797.9 & 6284.2 & 1.2460 & \\
\hline & [O II $]$ & 3726.8 & 8364.9 & 1.2446 & \\
\hline \multirow[t]{2}{*}{ IVS B0110-668 } & $\left.\mathrm{C}_{\mathrm{III}}\right]$ & 1908.7 & 4174.2 & 1.1869 & $1.1888 \pm 0.0021$ \\
\hline & Mg II & 2797.9 & 6129.1 & 1.1906 & \\
\hline \multirow[t]{3}{*}{ IVS B0221-171 } & $\mathrm{C}$ III] & 1908.7 & 3840.8 & 1.0122 & $1.0152 \pm 0.0017$ \\
\hline & Mg II & 2797.9 & 5639.3 & 1.0155 & \\
\hline & $\mathrm{H} \gamma$ & 4340.5 & 8758.9 & 1.0180 & \\
\hline \multirow[t]{7}{*}{ IVS B1505-304 } & Ly $\lim$ & 911.5 & 4031.2 & 3.4226 & $3.3684 \pm 0.0023$ \\
\hline & $\operatorname{Ly} \beta$ & 1025.7 & 4524.4 & 3.4110 & \\
\hline & $\operatorname{Ly} \alpha$ & 1215.7 & 5412.6 & 3.4524 & \\
\hline & $\mathrm{Nv}$ & 1240.1 & 5466.2 & 3.4077 & \\
\hline & Si IV & 1396.8 & 6095.4 & 3.3639 & \\
\hline & C IV & 1549.1 & 6770.2 & 3.3705 & \\
\hline & C III] & 1908.7 & 8342.4 & 3.3707 & \\
\hline \multirow[t]{4}{*}{ IVS B1511-476 } & CIV & 1549.1 & 3957.3 & 1.5546 & $1.5512 \pm 0.0018$ \\
\hline & He II & 1640.4 & 4185.6 & 1.5515 & \\
\hline & C III] & 1908.7 & 4859.9 & 1.5461 & \\
\hline & Mg II & 2797.9 & 7142.2 & 1.5527 & \\
\hline \multirow[t]{12}{*}{ IVS B1635-141 } & {$[\mathrm{Ne} \mathrm{v}]$} & 3345.4 & 4206.9 & 0.2575 & $0.2575 \pm 0.0004$ \\
\hline & {$[\mathrm{Ne} \mathrm{v}]$} & 3425.5 & 4315.8 & 0.2599 & \\
\hline & [O II $]$ & 3726.0 & 4682.7 & 0.2568 & \\
\hline & [Ne III] & 3869.1 & 4868.1 & 0.2582 & \\
\hline & [Ne III] & 3967.8 & 4987.0 & 0.2569 & \\
\hline & $\mathrm{H} \delta$ & 4101.7 & 5155.7 & 0.2570 & \\
\hline & $\mathrm{H} \gamma$ & 4340.5 & 5466.8 & 0.2595 & \\
\hline & $\mathrm{H} \beta$ & 4861.3 & 6111.1 & 0.2571 & \\
\hline & [O III $]$ & 4958.9 & 6229.7 & 0.2563 & \\
\hline & [O III $]$ & 5006.8 & 6289.7 & 0.2562 & \\
\hline & $\mathrm{H} \alpha$ & 6562.8 & 8233.8 & 0.2546 & \\
\hline & {$[\mathrm{N} \mathrm{II}]$} & 6583.5 & 8267.3 & 0.2558 & \\
\hline \multirow[t]{4}{*}{ IVS B1659-621 } & Si IV & 1396.8 & 3845.5 & 1.7532 & $1.7547 \pm 0.0012$ \\
\hline & C IV & 1549.1 & 4269.0 & 1.7559 & \\
\hline & $\mathrm{C}$ III] & 1908.7 & 5253.0 & 1.7521 & \\
\hline & Mg II & 2797.9 & 7716.0 & 1.7578 & \\
\hline \multirow[t]{3}{*}{ IVS B1707-038 } & C IV & 1549.1 & 4523.0 & 1.9199 & $1.9231 \pm 0.0017$ \\
\hline & C III] & 1908.7 & 5584.3 & 1.9256 & \\
\hline & Mg II & 2797.9 & 8180.6 & 1.9238 & \\
\hline \multirow[t]{7}{*}{ IVS B1726-038 } & Mg II & 2797.9 & 4647.3 & 0.6610 & $0.6617 \pm 0.0003$ \\
\hline & {$[\mathrm{Ne} \mathrm{v}]$} & 3345.4 & 5559.8 & 0.6619 & \\
\hline & {$[\mathrm{Ne} v]$} & 3425.5 & 5689.8 & 0.6610 & \\
\hline & [O II $]$ & 3726.8 & 6195.7 & 0.6625 & \\
\hline & [Ne III] & 3869.1 & 6428.5 & 0.6615 & \\
\hline & [Ne III] & 3967.8 & 6590.6 & 0.6610 & \\
\hline & $\mathrm{H} \gamma$ & 4340.5 & 7212.0 & 0.6616 & \\
\hline
\end{tabular}

Table 2

(Continued)

\begin{tabular}{|c|c|c|c|c|c|}
\hline Source & Line & $\begin{array}{c}\lambda_{\text {rest }} \\
(\AA)\end{array}$ & $\begin{array}{l}\lambda_{\text {obs }} \\
(\AA)^{\mathrm{a}}\end{array}$ & $z$ & Mean $z$ \\
\hline & $\mathrm{H} \beta$ & 4861.3 & 8077.8 & 0.6616 & \\
\hline & [O III] & 4958.9 & 8246.2 & 0.6629 & \\
\hline & [O III $]$ & 5006.8 & 8321.9 & 0.6621 & \\
\hline \multirow[t]{7}{*}{ IVS B1758-651 } & C III] & 1908.7 & 4199.1 & 1.2000 & $1.1991 \pm 0.0006$ \\
\hline & $\mathrm{C}$ II] & 2326.9 & 5117.0 & 1.1990 & \\
\hline & [Ne IV] & 2423.8 & 5327.0 & 1.1977 & \\
\hline & Mg II & 2797.9 & 6156.8 & 1.2005 & \\
\hline & {$[\mathrm{Ne} \mathrm{v}]$} & 3345.4 & 7331.9 & 1.1916 & \\
\hline & {$[\mathrm{Ne} \mathrm{v}]$} & 3425.5 & 7527.8 & 1.1976 & \\
\hline & {$[\mathrm{O} \mathrm{II}]$} & 3726.8 & 8197.9 & 1.1997 & \\
\hline \multirow[t]{4}{*}{ IVS B1815-553 } & CIV & 1549.1 & 4070.3 & 1.6276 & $1.6292 \pm 0.0013$ \\
\hline & $\left.\mathrm{C}_{\mathrm{III}}\right]$ & 1908.7 & 5016.8 & 1.6284 & \\
\hline & $\mathrm{C}$ II] & 2326.9 & 6123.7 & 1.6317 & \\
\hline & Mg II & 2797.9 & 7384.2 & 1.6391 & \\
\hline \multirow[t]{6}{*}{ IVS B1852-534 } & $\mathrm{Mg}_{\text {II }}$ & 2797.9 & 4973.4 & 0.7775 & $0.7779 \pm 0.0007$ \\
\hline & {$[\mathrm{O}$ II $]$} & 3726.8 & 6628.4 & 0.7786 & \\
\hline & $\mathrm{H} \delta$ & 4101.7 & 7280.6 & 0.7750 & \\
\hline & $\mathrm{H} \gamma$ & 4340.5 & 7715.9 & 0.7777 & \\
\hline & [O III $]$ & 4958.9 & 8830.3 & 0.7807 & \\
\hline & [O III $]$ & 5006.8 & 8900.6 & 0.7777 & \\
\hline \multirow[t]{5}{*}{ IVS B1928-698 } & C IV & 1549.1 & 3836.7 & 1.4768 & $1.4807 \pm 0.0020$ \\
\hline & C III] & 1908.7 & 4730.3 & 1.4782 & \\
\hline & $\mathrm{C}$ II] & 2326.9 & 5755.2 & 1.4733 & \\
\hline & Mg II & 2797.9 & 6952.9 & 1.4850 & \\
\hline & {$[\mathrm{Ne} \mathrm{v}]$} & 3345.4 & 8306.2 & 1.4829 & \\
\hline \multirow{5}{*}{ IVS B2107-105 } & $\operatorname{Ly} \alpha$ & 1215.7 & 4258.3 & 2.5028 & $2.5004 \pm 0.0012$ \\
\hline & $\mathrm{Nv}$ & 1240.1 & 4347.6 & 2.5057 & \\
\hline & Si IV & 1396.8 & 4889.9 & 2.5009 & \\
\hline & C IV & 1549.1 & 5418.5 & 2.4979 & \\
\hline & $\left.\mathrm{C}_{\mathrm{III}}\right]$ & 1908.7 & 6680.4 & 2.4999 & \\
\hline \multirow[t]{5}{*}{ IVS B2117-614 } & $\mathrm{C}$ III] & 1908.7 & 3848.3 & 1.0161 & $1.0168 \pm 0.0011$ \\
\hline & $\mathrm{C}$ II] & 2326.9 & 4685.5 & 1.0136 & \\
\hline & Mg II & 2797.9 & 5651.2 & 1.0198 & \\
\hline & {$[\mathrm{O}$ II $]$} & 3726.8 & 7514.2 & 1.0163 & \\
\hline & $\mathrm{H} \gamma$ & 4340.5 & 8760.6 & 1.0184 & \\
\hline \multirow[t]{11}{*}{ IVS B2135-184 } & [O II $]$ & 3727.7 & 4430.9 & 0.1887 & $0.1887 \pm 0.0001$ \\
\hline & [Ne III] & 3869.1 & 4598.4 & 0.1885 & \\
\hline & $\mathrm{Ca} \mathrm{H}$ & 3934.8 & 4680.8 & 0.1896 & \\
\hline & $\mathrm{Ca} \mathrm{K}$ & 3969.6 & 4718.1 & 0.1886 & \\
\hline & $\mathrm{H} \gamma$ & 4340.5 & 5158.2 & 0.1884 & \\
\hline & $\mathrm{H} \beta$ & 4861.3 & 5778.3 & 0.1886 & \\
\hline & [O III] & 4958.9 & 5889.4 & 0.1876 & \\
\hline & [O III] & 5006.8 & 5949.7 & 0.1883 & \\
\hline & $\mathrm{Na} \mathrm{D}$ & 5892.9 & 7005.7 & 0.1888 & \\
\hline & $\mathrm{H} \alpha$ & 6562.8 & 7805.7 & 0.1894 & \\
\hline & {$[\mathrm{S}$ II $]$} & 6716.4 & 7986.3 & 0.1891 & \\
\hline \multirow[t]{2}{*}{ IVS B2158-167 } & Mg II & 2797.9 & 5142.3 & 0.8379 & $0.8355 \pm 0.0024$ \\
\hline & [O III] & 5006.8 & 9178.2 & 0.8331 & \\
\hline \multirow[t]{6}{*}{ IVS B2211-388 } & [O II $]$ & 3727.7 & 5180.5 & 0.3897 & $0.3888 \pm 0.0004$ \\
\hline & $\mathrm{H} \beta$ & 4861.3 & 6747.3 & 0.3880 & \\
\hline & [O III] & 4958.9 & 6886.8 & 0.3888 & \\
\hline & [O III] & 5006.8 & 6954.6 & 0.3890 & \\
\hline & $\mathrm{H} \alpha$ & 6562.8 & 9108.5 & 0.3879 & \\
\hline & {$[\mathrm{N}$ II $]$} & 6583.5 & 9140.3 & 0.3884 & \\
\hline \multirow[t]{4}{*}{ IVS B2220-163 } & $\left.\mathrm{C}_{\mathrm{II}}\right]$ & 2326.9 & 4385.4 & 0.8846 & $0.8811 \pm 0.0002$ \\
\hline & Mg II & 2797.9 & 5262.7 & 0.8809 & \\
\hline & [O II $]$ & 3726.8 & 7011.9 & 0.8815 & \\
\hline & [Ne III] & 3869.1 & 7277.4 & 0.8809 & \\
\hline \multirow[t]{2}{*}{ IVS B2234-253 } & C III] & 1908.7 & 4347.3 & 1.2776 & $1.2788 \pm 0.0011$ \\
\hline & Mg II & 2797.9 & 6379.3 & 1.2800 & \\
\hline \multirow[t]{3}{*}{ IVS B2236-572 } & Mg II & 2797.9 & 4381.2 & 0.5659 & $0.5686 \pm 0.0023$ \\
\hline & {$[\mathrm{Ne} \mathrm{v}]$} & 3425.5 & 5388.8 & 0.5731 & \\
\hline & [O II $]$ & 3726.8 & 5838.7 & 0.5667 & \\
\hline \multirow[t]{2}{*}{ IVS B2239-631 } & Mg II & 2797.9 & 3901.5 & 0.3944 & $0.3924 \pm 0.0005$ \\
\hline & {$[\mathrm{Ne} \mathrm{V}]$} & 3345.4 & 4649.6 & 0.3898 & \\
\hline
\end{tabular}


Table 2

(Continued)

\begin{tabular}{|c|c|c|c|c|c|}
\hline Source & Line & $\begin{array}{c}\lambda_{\text {rest }} \\
(\AA)\end{array}$ & $\begin{array}{l}\lambda_{\text {obs }} \\
(\AA)^{\mathrm{a}}\end{array}$ & $z$ & Mean $z$ \\
\hline & {$[\mathrm{Ne} \mathrm{v}]$} & 3425.5 & 4774.9 & 0.3939 & \\
\hline & [Ne III] & 3869.1 & 5385.5 & 0.3919 & \\
\hline & $\mathrm{H} \gamma$ & 4340.5 & 6056.1 & 0.3953 & \\
\hline & $\mathrm{H} \beta$ & 4861.3 & 6767.9 & 0.3922 & \\
\hline & [O III] & 4958.9 & 6899.8 & 0.3914 & \\
\hline & [O III] & 5006.8 & 6968.1 & 0.3917 & \\
\hline & $\mathrm{H} \alpha$ & 6562.8 & 9129.7 & 0.3911 & \\
\hline \multirow[t]{3}{*}{ IVS B2318-087 } & $\operatorname{Ly} \alpha$ & 1215.7 & 5065.5 & 3.1668 & $3.1639 \pm 0.0033$ \\
\hline & C IV & 1549.1 & 6445.6 & 3.1610 & \\
\hline & C III] & 1908.7 & 7976.2 & 3.1788 & \\
\hline \multirow[t]{6}{*}{ IVS B2321-065 } & $\operatorname{Ly} \alpha$ & 1215.7 & 3820.9 & 2.1430 & $2.1440 \pm 0.0020$ \\
\hline & $\mathrm{Nv}$ & 1240.1 & 3901.7 & 2.1462 & \\
\hline & Si IV & 1396.8 & 4414.6 & 2.1606 & \\
\hline & C IV & 1549.1 & 4865.6 & 2.1410 & \\
\hline & $\mathrm{C}$ III] & 1908.7 & 5970.9 & 2.1282 & \\
\hline & Mg II & 2797.9 & 8807.9 & 2.1480 & \\
\hline \multirow[t]{5}{*}{ IVS B2327-459 } & Mg II & 2797.9 & 4051.8 & 0.4482 & $0.4471 \pm 0.0004$ \\
\hline & [O II $]$ & 3726.8 & 5392.7 & 0.4470 & \\
\hline & $\mathrm{H} \beta$ & 4861.3 & 7033.7 & 0.4469 & \\
\hline & [O III] & 4958.9 & 7169.0 & 0.4457 & \\
\hline & [O III] & 5006.8 & 7242.4 & 0.4465 & \\
\hline \multirow[t]{4}{*}{ IVS B2344-514 } & Si IV & 1396.8 & 3873.2 & 1.7730 & $1.7502 \pm 0.0060$ \\
\hline & C IV & 1549.1 & 4250.8 & 1.7441 & \\
\hline & C III] & 1908.7 & 5261.0 & 1.7563 & \\
\hline & Mg II & 2797.9 & 7700.3 & 1.7521 & \\
\hline \multirow[t]{3}{*}{ IVS B2354-251 } & CIV & 1549.1 & 4045.2 & 1.6114 & $1.6137 \pm 0.0014$ \\
\hline & C III] & 1908.7 & 4992.4 & 1.6156 & \\
\hline & Mg II & 2797.9 & 7314.0 & 1.6141 & \\
\hline
\end{tabular}

Note. ${ }^{\text {a }}$ A colon after the observed wavelength indicates that the line position was uncertain because of blending, proximity to a noise spike, or associated absorption, and hence was not used to determine the redshift.

redshift of $z=0.7804 \pm 0.0013$ is based on a much higher $\mathrm{S} / \mathrm{N}$ spectrum.

2. IVS B0008-300-strong absorption feature blueward of the Ly $\alpha$ emission line.

3. IVS B0028-396 - only two lines were identified in a noisy spectrum but the redshift is secure.

4. IVS B0055-059-reported as a quasar with redshift $z=$ $1.23998(Q=2)$ in the $6 \mathrm{dF}$ survey (Jones et al. 2009); our redshift of $z=1.2456 \pm 0.0008$ is more reliable because of the higher $\mathrm{S} / \mathrm{N}$ spectrum.

5. IVS B1707-038-previously known as a BL Lac object (Halpern et al. 2003), but weak emission lines were clearly detected in our spectrum at $z=1.9231$.

6. IVS B1505-304-the highest redshift object, $z=3.40$, in this sample; strong absorption shortward of Ly $\alpha$, with Lyman limit absorption at or close to the emission redshift.

7. IVS B2135-184-original galaxy identification by Gearhart et al. (1972).

8. IVS B2318-087-Faint object, but clear emission lines of $\operatorname{Ly} \alpha$ and C IV give us confidence that our redshift of $z=3.1639$ is correct.

9. IVS B2344-514-ICRF2 defining source. The redshift $z=2.67$ cited by Fey et al. (2009) is not correct, possibly due to the misidentification of C IV with $\operatorname{Ly} \alpha$.

\subsection{IVS B1923+210}

The optical counterpart (see Figure 2, object 3) was previously observed at the Russian 6 m telescope (Maslennikov et al. 2010), and no emission lines were found. For the current campaign we observed all three optical objects located close to the ICRF2 position at $19^{\mathrm{h}} 25^{\mathrm{m}} 59^{\mathrm{s}} .605+21^{\circ} 06^{\prime} 26^{\prime \prime} .16(\mathrm{~J} 2000)$. The spectra of both objects 1 and 2 show the Ca II triplet at rest, which confirms them as stars. Object 3, which has $R \sim 20$ and is coincident with the radio position, shows no stellar absorption features although the $\mathrm{S} / \mathrm{N}$ is poor. There are also no obvious emission lines. The combination of a radio-optical coincidence and featureless spectrum is sufficient to suggest this as a probable BL Lac object.

\subsection{IVS B2300-307}

The object closest to the Tidbinbilla interferometer position was reported as a star of magnitude 16 by Jauncey et al. (1982) despite close positional agreement. Later radio observations confirmed it as a strong source $\sim 0.5 \mathrm{Jy}$ with a flat spectrum (Wright et al. 1991; Quiniento \& Cersosimo 1993; Reynolds et al. 1994). A VLBI image at $8.4 \mathrm{GHz}$ published by Ojha et al. (2005) reveals the classical double structure of an extragalactic radio source, with components separated by $r=3.3$ mas in positional angle $124^{\circ}$. Optical observations reveal a single object (Figure 3) with a stellar absorption spectrum characteristic of a $\mathrm{G}$ star. This star is offset by 3.7 arcsec from the ICRF2 radio position. The VLBI radio structure of IVS B2300-307 (Ojha

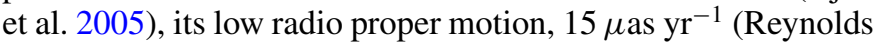
et al. 1994, IVS data from 2002), and its high brightness temperature, $10^{11} \mathrm{~K}$ (Preston et al. 1985), all strongly support its extragalactic nature. Therefore, we conclude that the field of IVS B2300-307 is obscured by the foreground star. Since there is no sign of an extra image at the precise ICRF2 position, marked with a cross in Figure 3, we estimate that the optical counterpart probably has $B \geqslant 21$.

\section{COMPARISON OF RADIO AND OPTICAL POSITIONS}

Correct identification of extragalactic radio sources can also be verified by comparison of their optical and radio coordinates. Along the Galactic plane the high dust absorption and the high density of Galactic stars can make identification impossible. In our case we compared radio coordinates from ICRF2 and optical coordinates from the SuperCOSMOS survey (Hambly et al. 2001). Of the 31 extragalactic sources, positions for only 25 could be accurately determined; the remainder were excluded, either because the images were too faint to be measured reliably by SuperCOSMOS or were blended because of low Galactic latitude. Two low-redshift objects (IVS B2135-184 and B2211-388) were excluded because their images were clearly non-stellar. We found the mean difference between the radio and optical positions to be $0.064 \pm 0$. ..021 with $1 \sigma \mathrm{rms}$ of 0.103 in right ascension and $0.023 \pm 0.031$ with $1 \sigma \mathrm{rms}$ of 0 .'155 in declination. The scatter in both coordinates is small and supports the claimed accuracy of SuperCOSMOS (Hambly et al. 2001).

While the mean difference in declination is negligible, the difference in right ascension marginally exceeds the $3 \sigma$ level. It is unlikely that a systematic shift between the radio and optical positions is due to physical reasons. Instead, this may be caused by a small rotation of the SuperCOSMOS right ascension reference point with respect to the ICRF2 origin. 
Table 3

Comparison between Radio and Optical Positions for the Stellar Misidentifications

\begin{tabular}{|c|c|c|c|c|c|c|c|}
\hline \multirow[t]{2}{*}{ Source } & \multicolumn{2}{|c|}{$\begin{array}{c}\text { ICRF2 Position } \\
\text { J2000 }\end{array}$} & \multicolumn{2}{|c|}{$\begin{array}{c}\text { Optical Position } \\
\text { J2000 }\end{array}$} & \multicolumn{2}{|c|}{$\begin{array}{lr}\Delta \alpha & \Delta \delta \\
\text { ICRF2-optical }\end{array}$} & \multirow{2}{*}{$\begin{array}{c}\sigma_{\mathrm{opt}} \\
\left(^{\prime \prime}\right)\end{array}$} \\
\hline & $(\mathrm{h} \mathrm{m} \mathrm{s})$ & $\left(0^{\prime \prime \prime \prime}\right)$ & $(\mathrm{s})$ & $\left({ }^{\prime \prime}\right)$ & $\left({ }^{\prime \prime}\right)$ & $\left({ }^{\prime \prime}\right)$ & \\
\hline IVS B1748-253 & 175151.263 & -252400.06 & 51.104 & 01.44 & 2.15 & 1.38 & 0.29 \\
\hline IVS B1822-173 & 182536.532 & -171849.85 & 36.566 & 50.57 & -0.49 & 0.72 & 0.21 \\
\hline IVS B1923+210 & 192559.605 & +210626.16 & 59.662 & 24.37 & -0.80 & -1.79 & 0.22 \\
\hline IVS B2300-307 & 230305.821 & -303011.47 & 05.897 & 15.06 & -0.98 & 3.59 & 0.15 \\
\hline
\end{tabular}

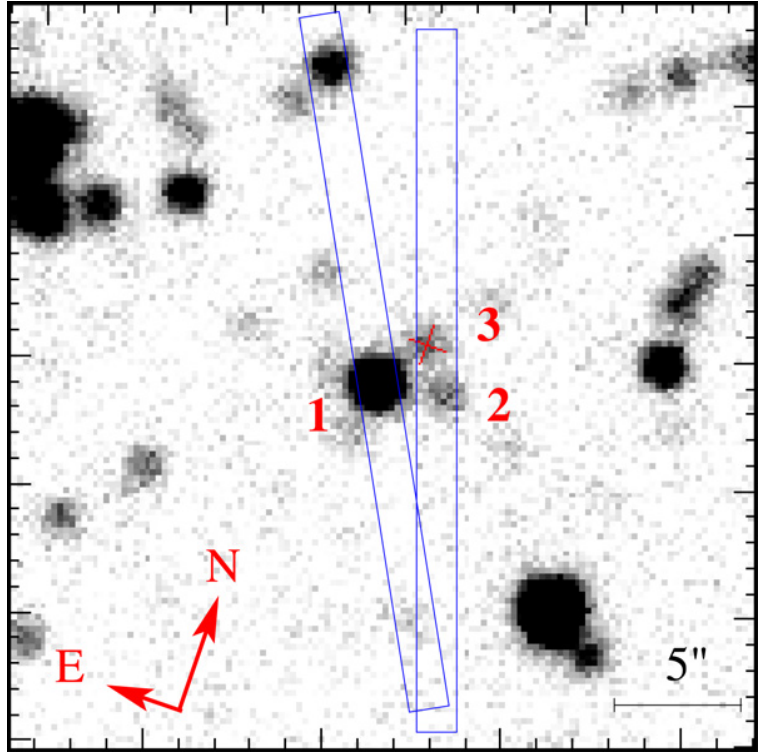

Figure 2. NTT acquisition image of the area near the radio position of the source IVS B1923+210. The optical identification is object 3, marked with a red cross; objects 1 and 2 showed stellar spectra. The two slit positions used are marked by blue boxes.

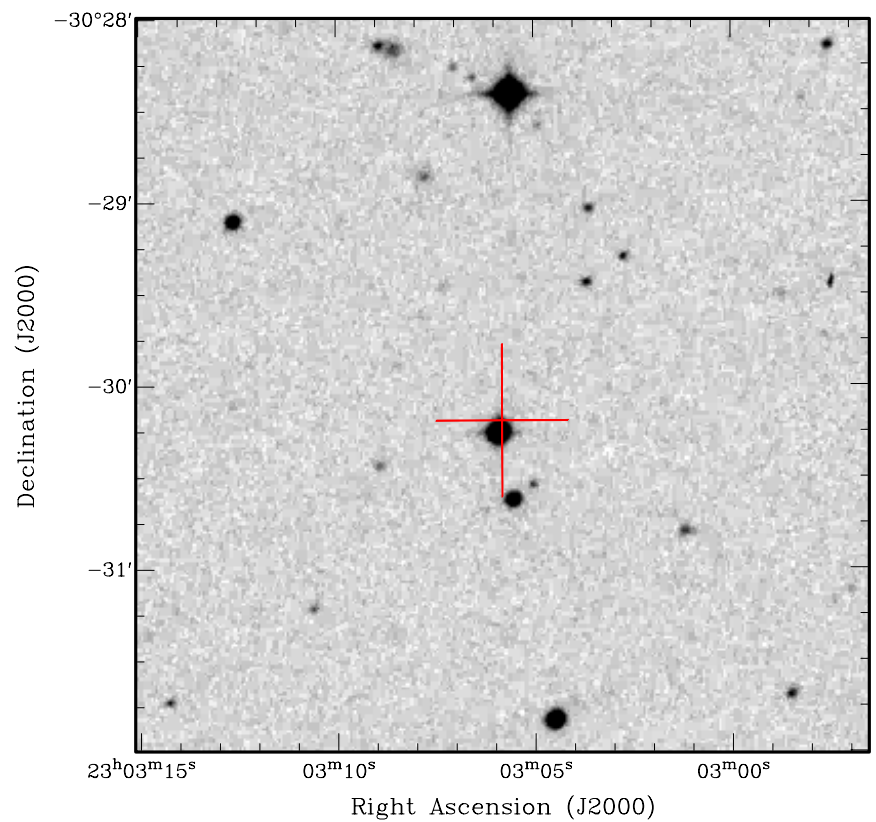

Figure 3. Finding chart for the source IVS B2300-307 from the UK Schmidt B image. The IVS position is marked with a cross, showing how the optical field of the radio source is obscured by the foreground star.

A similar radio-optical comparison was carried out for seven of the eight probable BL Lac objects, excluding
IVS B1923 + 210 which was too faint to be seen with SuperCOSMOS. The mean differences were not significant, with a $1 \sigma$ scatter of 0.15 in each coordinate. This result supports the identifications and hence their classification as BL Lac objects.

\section{SUMMARY AND CONCLUSION}

We performed spectroscopic observations of the optical counterparts of 47 southern radio sources from the ICRF and measured 31 new redshifts, with two of them having $z \geqslant 3$. At least two emission lines were confidently identified for each target. Redshifts were determined principally from isolated lines, with lines that were weak, blended, or affected by strong absorption given lower (or zero) weight. Eight targets showed featureless spectra and were considered BL Lac objects.

There were eight ICRF2 defining radio sources in this run. Five of them-IVS B1659-621, B1758-651, B1815-553, B2236-572, and B2344-514-were confirmed as extragalactic sources. Source IVS B1631-810 was confirmed as a BL Lac object with a featureless spectrum, but two other sources, IVS B0107-610 and IVS B1443-162, were observed in poor weather conditions, and no emission lines could be confidently identified in their spectra.

This paper is based on observations collected at the European Organisation for Astronomical Research in the Southern Hemisphere, Chile, programme 058.A-0855(A). Two of us, Titov and Jauncey, were supported by a travel grant from the Australian Nuclear Science Technology Organisation (ANSTO) in their access to Major Research Facilities Program (AMRFP, reference number AMRFP 10/11-O-04) to travel to La Silla.

Facility: NTT (GMOS)

\section{REFERENCES}

Bourda, G., Charlot, P., \& Le Campion, J.-F. 2008, A\&A, 490, 403

Bourda, G., Collioud, A., Charlot, P., Porcas, R., \& Garrington, S. 2011, A\&A, 526, A102

Fey, A., Gordon, G., \& Jacobs, C. (ed.) 2009, The Second Realization of the International Celestial Reference Frame by Very Long Baseline Interferometry, IERS Technical Notes 35

Gearhart, M. R., Lund, J., Frantz, D., \& Kraus, J. 1972, AJ, 77, 557

Halpern, J. P., Erakleous, M., \& Matrox, J. R. 2003, AJ, 125, 573

Hambly, N. C., Davenhall, A. C., Irwin, M. J., \& MacGillivray, H. T. 2001, MNRAS, 326, 1315

Jauncey, L. D., Batty, M. J., Gulkis, S., \& Savage, A. 1982, AJ, 87, 763

Jones, D. H., Read, M. A., Saunders, W., et al. 2009, MNRAS, 399, 683

Lindegren, G., Babusiaux, C., Bailer-Jones, C., et al. 2008, in IAU Symp. 248, A Giant Step: From Milli- to Micro-arcsecond Astrometry, ed. W. J. Wenjin, I. Platais, \& M. A. C. Perryman (Cambridge: Cambridge Univ. Press), 217

Ma, C., Arias, E. F., Eubanks, T. M., et al. 1998, AJ, 116, 516

Maslennikov, K., Boldycheva, A., Malkin, Z., \& Titov, O. 2010, Astrophys., 53, 147 
McCarthy, D., \& Petit, G. (ed.) 2004, IERS Conventions (2003), IERS Technical Notes 32 (Frankfurt am Main: Verlad des Bundesamts für Kartographie und Geodäsie)

Ojha, R., Fey, A. L., Charlot, P., et al. 2005, AJ, 130, 2529

Perryman, M. A. C., de Boer, K. S., Gilmore, G., et al. 2001, A\&A, 369, 339

Preston, R. A., Morabito, D. D., Williams, J. G., et al. 1985, AJ, 90, 1599
Quiniento, Z. M., \& Cersosimo, J. C. 1993, A\&AS, 97, 435

Reynolds, J. E., Jauncey, D. L., Russell, J. L., et al. 1994, AJ, 108, 725

Schlüter, W., \& Behrend, D. 2007, JG, 81, 379

Titov, O., \& Malkin, Z. 2009, A\&A, 506, 1477

Wright, A. E., Wark, R. M., Troup, E., et al. 1991, MNRAS, 251, 330 